\title{
Hokkaido-wide eDNA Monitoring for Sakhalin Taimen, Endangered Salmonid Species
}

\author{
Hiroki Mizumoto ${ }^{1,2}$ and Hitoshi Araki ${ }^{1}$ \\ ${ }^{1}$ Research Faculty of Agriculture, Hokkaido University, Kita-9 Nishi-9 Kita-ku, Sapporo 060-8589, Hokkaido, \\ Japan
}

${ }^{2}$ Salmon Research Department, Fisheries Resources Institute, Japan Fisheries Research and Education Agency, 2-41, Nakanoshima 2-jo, Toyohira-ku, Sapporo, 062-0922, Hokkaido, Japan

Keywords: Sakhalin taimen, environmental DNA, endangered species, distribution

Sakhalin taimen (Parahucho perryi) is one of the largest anadromous salmonid fish species in the world. While they are known as a keystone species in river ecosystems, they are currently listed as a critically endangered species on the IUCN Red List due to human disturbances which have caused habitat degradations (e.g., deforestation and river reconstruction) (Fukushima 2006; Rand 2006; Fukushima et al. 2007; Fukushima et al. 2011). For now, their distribution and population status are unclear because it is very difficult to find the organisms in natural environments - just like many other endangered species. In this study, we estimated their current distribution and biomass simultaneously in Hokkaido, Japan, using an environmental DNA (eDNA) species-specific detection system established in a previous study (Mizumoto et al. 2018). We collected eDNA samples from 120 river systems and applied this detection system to them. Our results suggest that distribution of Sakhalin taimen is quite limited and that they are at the brink of extinction for most of the river systems in Hokkaido. Furthermore, we found low levels of human disturbances, especially on river connectivity in two major river systems, where high eDNA concentrations of Sakhalin taimen were detected. These results suggest that eDNA methods play an important role in understanding the population status of endangered species in aquatic environments, and that river consecutiveness is critical for the sustainability of this long-living, precious anadromous fish species. See Mizumoto et al. (2020) for details.

\section{REFERENCES}

Fukushima, M. 2006. The effects of damming on masu salmon and the Sakhalin taimen and the assessment of their conservation areas based on predictive habitat models. Ecol. Civ. Eng. 8: 233-244.

Fukushima, M., S. Kameyama, M. Kaneko, K. Nakao, and S.E. Ashley. 2007. Modelling the effects of dams on freshwater fish distributions in Hokkaido, Japan. Freshw. Biol. 52: 1511-1524.

Fukushima, M., H. Shimazaki, P.S. Rand, and M. Kaeriyama. 2011. Reconstructing Sakhalin taimen Parahucho perryi historical distribution and identifying causes for local extinctions. Trans. Am. Fish. Soc. 140: 1-13.

Mizumoto, H., H. Urabe, T. Kanbe, M. Fukushima, and H. Araki. 2018. Establishing an environmental DNA method to detect and estimate the biomass of Sakhalin taimen, a critically endangered Asian salmonid. Limnology 19: 219-227.

Mizumoto, H., T. Mitsuzuka, and H. Araki. 2020. An Environmental DNA Survey on Distribution of an Endangered Salmonid Species, Parahucho perryi, in Hokkaido, Japan. Front. Ecol. Evol. 8. 569425. doi: 10.3389/fevo.2020.569425.

Rand, P.S. 2006. Hucho perryi: IUCN (International Union for the Conservation of Nature) 2020 red list of threatened species, version 2020-1. IUCN. (Available at https://www.iucnredlist.org/species/61333/12462795) 